\title{
Towards the Quark-Gluon-Plasma
}

\author{
Johanna Stachel \\ Physikalisches Institut der Universität Heidelberg, 69120 Heidelberg, Germany
}

\section{INTRODUCTION}

A new phase of hadronic matter is expected to form at high temperature and/or high baryon density. This is based on considerations in perturbative quantum chromo dynamics (QCD) and in chiral perturbation theory. The most quantitative information about where this transition is expected to happen comes from numerical solutions of QCD on the lattice where large progress was made during the past few years [1]. The systematic influence of the size of the space-time lattice, of the quark masses, and of the way to implement fermions on the lattice has been explored. Extrapolations to the chiral limit of a vanishing mass of the pseudoscalar Goldstone boson, the pion, give a critical temperature of $20 \%$ of the $\rho$ mass, i.e. $154 \mathrm{MeV}$ [2, 3]. The calculations indicate that at this temperature deconfinement is lifted and the quark condensate disappears leading to the restoration of chiral symmetry. The quark-gluon plasma (QGP) is formed. This is associated with a rapid rise in the number of degrees of freedom, and therefore the energy and entropy density, by typically one order of magnitude over a temperature interval of the order of $10 \mathrm{MeV}$.

This quark-gluon plasma presumably existed in the early universe until it hadronized at an age of ten microseconds and it may exist today in the interior of neutron stars. In order to produce this new phase in experiments in the laboratory collisions of heavy nuclei at the highest possible energies are considered the most likely tool. An experimental program started at the Brookhaven AGS and at the CERN SPS in 1986 and since 1992 and 1994, respectively, collisions of the heaviest nuclei are studied.

For heating and population of new degrees of freedom in the nuclear fireball the relevant number is the energy that is available in the c.m. system (without counting the masses of the constituent nucleons). For $\mathrm{Au}+\mathrm{Au}$ collisions at the AGS and for $\mathrm{Pb}+\mathrm{Pb}$ collisions at the SPS the respective numbers are 600 and $3200 \mathrm{GeV}$ per collision. After the fireball hadronizes these numbers materialize in terms of about 900 and 2400 hadrons in the final state. It is interesting to note that the two accelerators give access to two quite different regimes in the final state: at the AGS the number of nucleons and pions is about comparable while at the SPS pions dominate by a factor of six. In either case, the nucleons are dominantly the constituent nucleons of projectile and target.

In the following the experimental results available after one decade of studying ultra relativistic heavy ion collisions will be reviewed and discussed vis-a-vis the expected phase transition to the QGP. 


\section{EQUILIBRATION}

One of the key questions is the issue whether the fireball ever reaches equilibrium during its evolution. For the hadronic final state this question can be addressed in terms of asking whether the hadron yields are consistent with expectations for a statistical ensemble.

In Figure 1 is shown the systematics of relative abundances of produced hadrons from the AGS after integrating over longitudinal and transverse momenta as much as the data allow. In this way the analysis becomes independent of dynamic effects such as expansion. The relative abundances span about 9 orders of magnitude comparing pion and nucleon yields to those of antideuterons. The main difference to results from nucleon nucleon collisions is a significant enhancement of strangeness, e.g. of a factor two to three in the kaon yield relative to pions in central $\mathrm{Au}+\mathrm{Au}$ collisions. Central $\mathrm{Si}+\mathrm{Au}$ and $\mathrm{Au}+\mathrm{Au}$ collisions lead to very similar results.

The experimental data are compared to calculations [5] for a grand canonical ensemble and there are two free parameters adjusted to the data: a temperature $\mathrm{T}$ which is found to be between 120 and $140 \mathrm{MeV}$ and a baryochemical potential $\mu_{b}=540 \mathrm{MeV}$ driven by the pion to nucleon ratio. One can see that the data are rather well described by this simple assumption.

This observation of enhanced strangeness production becomes even more dramatic in central $\mathrm{Pb}+\mathrm{Pb}$ collisions at the SPS where also multiply strange baryons have been studied. Figure 2 shows for semicentral to central $\mathrm{Pb}+\mathrm{Pb}$ collisions an enhancement for

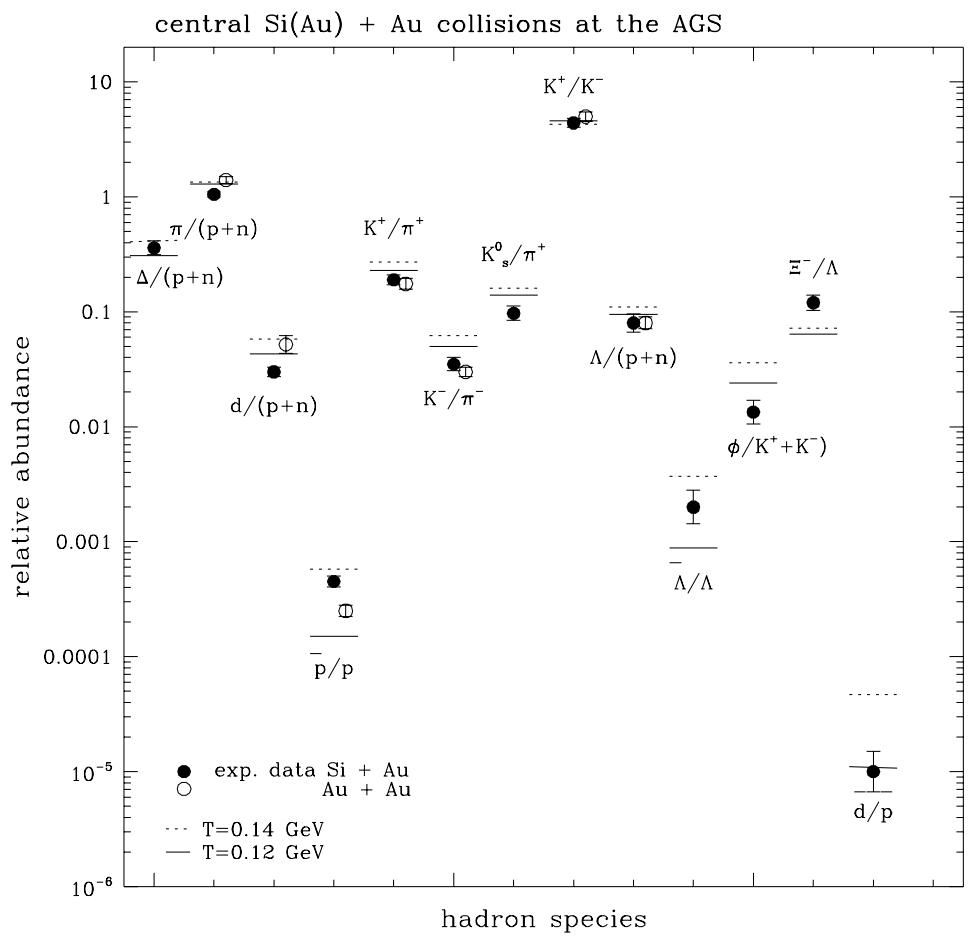

Figure 1. Hadron yields for central 14.6 A GeV/c Si+Au and $11 \mathrm{~A} \mathrm{GeV/c} \mathrm{Au+Au} \mathrm{collisions}$ at the AGS compared to a thermal model calculation for two temperatures. Figure from 4 . 


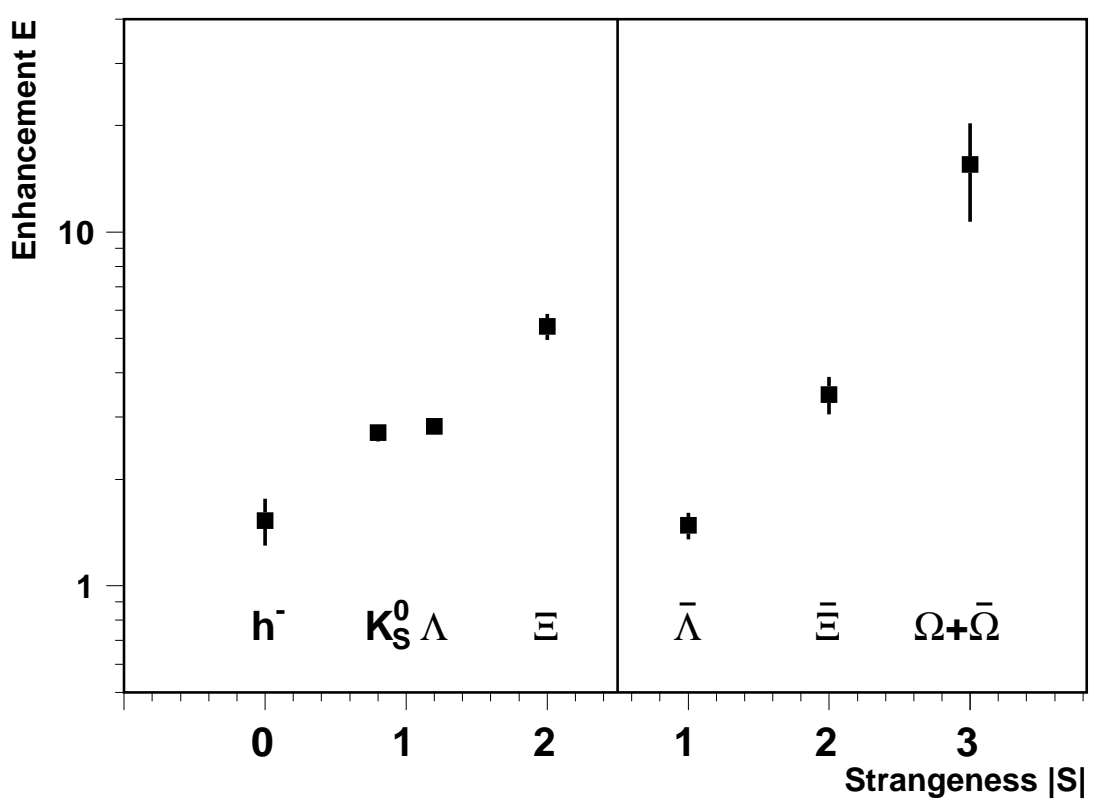

Figure 2. Enhancement of strange particle production in mid-central to central $158 \mathrm{~A} \mathrm{GeV} / \mathrm{c}$ $\mathrm{Pb}+\mathrm{Pb}$ collisions relative to $\mathrm{p}+\mathrm{Au}$ collisions. Figure from [6].

hadrons carrying strangeness rising dramatically with increasing strangeness. Here the enhancement is defined by scaling the yield of various produced particles to the number of nucleons participating in the collision, as defined by the impact parameter, and normalizing the result for $\mathrm{p}+\mathrm{Pb}$ collisions to unity. The triply strange omega baryons are produced about a factor 15 more frequently than expected for a simple linear scaling with the number of participating nucleons.

A summary of the yields of produced particles for central $\mathrm{Pb}+\mathrm{Pb}$ collisions at the SPS is shown in Figure 3. This combines data from all heavy ion experiments. Again the experimental data are compared to a calculation for a grand canonical ensemble, including the know hadron spectrum up to 1.5 and $2 \mathrm{GeV} / \mathrm{c}^{2}$ for mesons and baryons. And again the agreement is very good. The resulting two fit parameters are $\mathrm{T}=170 \mathrm{MeV}$ and $\mu_{b}$ $=280 \mathrm{MeV}$. The higher temperature and lower baryochemical potential as compared to the AGS fit are reflected in a much smaller dynamic range of yields of produced particles spanning in this case only about 4 orders of magnitude between pion and antideuteron. There is no indication that strangeness is suppressed vis-a-vis the equilibrium assumption and this is in marked contrast to nucleon-nucleon collisions where a similar analysis is only successful if one allows for explicit suppression by about a factor of two of particles carrying strangeness 8 .

In summary, one can conclude that hadron yields at AGS and SPS are consistent with the assumption of equilibration in terms of produced particle species. The values of temperature and baryochemical potential resulting from the fit with a statistical ensemble thus determine the points in the phase diagram when, during the evolution of the fireball, the hadron yields are frozen. This happens when inelastic collisions cease and this point is termed 'chemical freeze-out'. In order to put these freeze-out points into perspective relative to the expected phase transition, the result for the critical point from lattice QCD 
has to be extended towards finite baryon density. This can be done by constructing the phase boundary between a complete hadron gas as used e.g. in the yield calculations and an ideal gas of quarks and gluons using the Gibbs' conditions. Such a calculation [9] is shown in Figure 4 as the hatched band in comparison to the freeze-out points determined from yield ratios. There, the lower boundary of the hatched band reproduces the currently best result from lattice QCD at zero baryon density, the upper edge reflects the maximum systematic error still possibly contained in these calculations [12].

One notices that the chemical freeze-out points at the AGS and SPS are near or at the phase boundary while the points from the lower SIS energy show that there the system is far from the critical curve. It was noted recently [13] that the empirical curve outlined by the experimental points corresponds to a constant energy per hadron of about $1 \mathrm{GeV}$.

The question of 'thermal freeze-out' or the point when also elastic collisions seize can be addressed by inspecting the hadron spectra. Again, there is a distinct difference to the behavior know from $\mathrm{p}+\mathrm{p}$ collisions. Both at the AGS and SPS slopes of distributions in transverse mass $m_{t}=\sqrt{p_{t}^{2}+m^{2}}$ rise significantly with increasing mass. This is shown for the case of central $\mathrm{Pb}+\mathrm{Pb}$ collisions at the SPS in Figure 5 combining data from the different experiments. The dotted line indicates the approximate behavior.

Such an ordering in mass arises when the random thermal motion is superimposed to e.g. a common transverse expansion of the system [18] and such a picture has already successfully accounted for the spectra from Si and S induced reactions at the AGS and SPS [5, 19]. However it was noted that a range of combinations of temperatures and appropriate expansion velocities can account for the experimental data [20]. This ambiguity can be avoided by choosing a certain initial condition and an equation of state; then for every

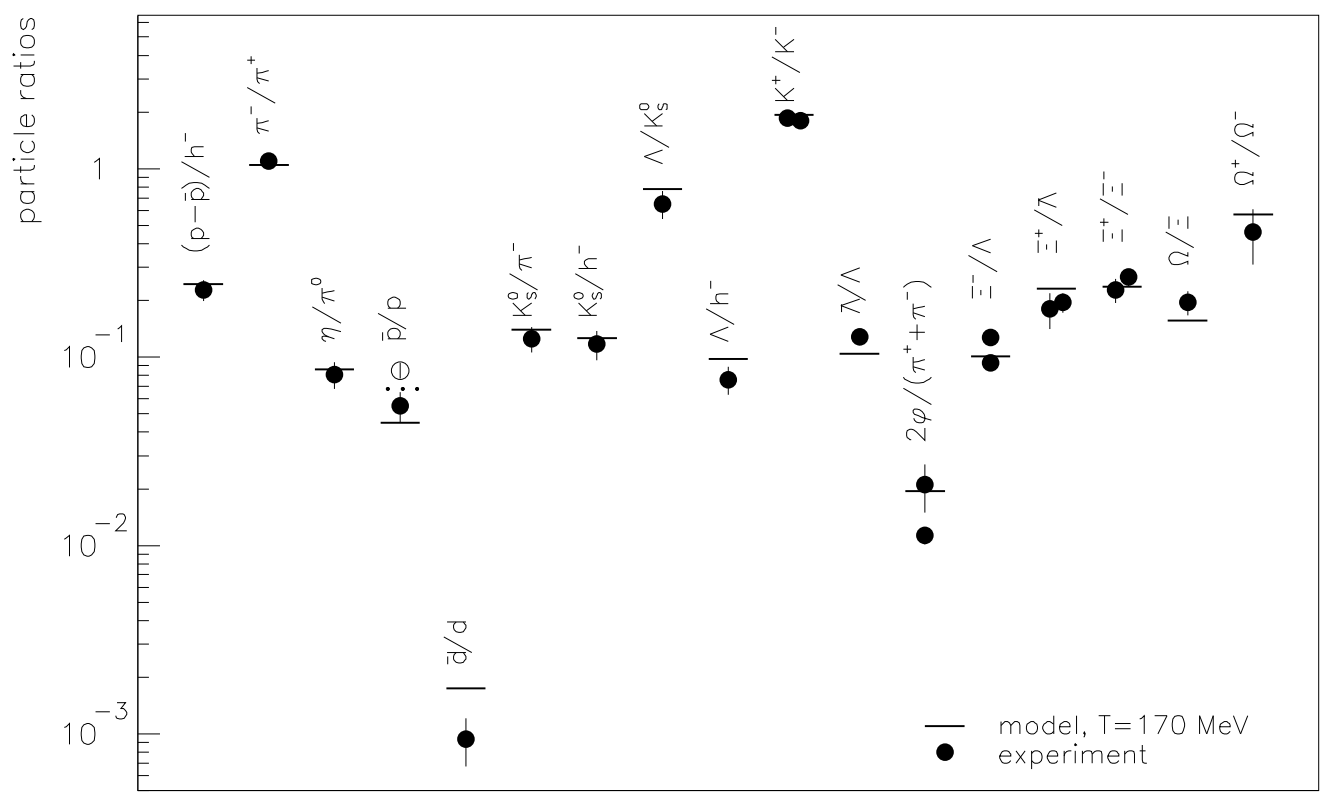

Figure 3. Hadron yields for central $\mathrm{Pb}+\mathrm{Pb}$ collisions at $158 \mathrm{~A} \mathrm{GeV} / \mathrm{c}$ at the SPS compared to a fit with a thermal model; Figure from [7]. 


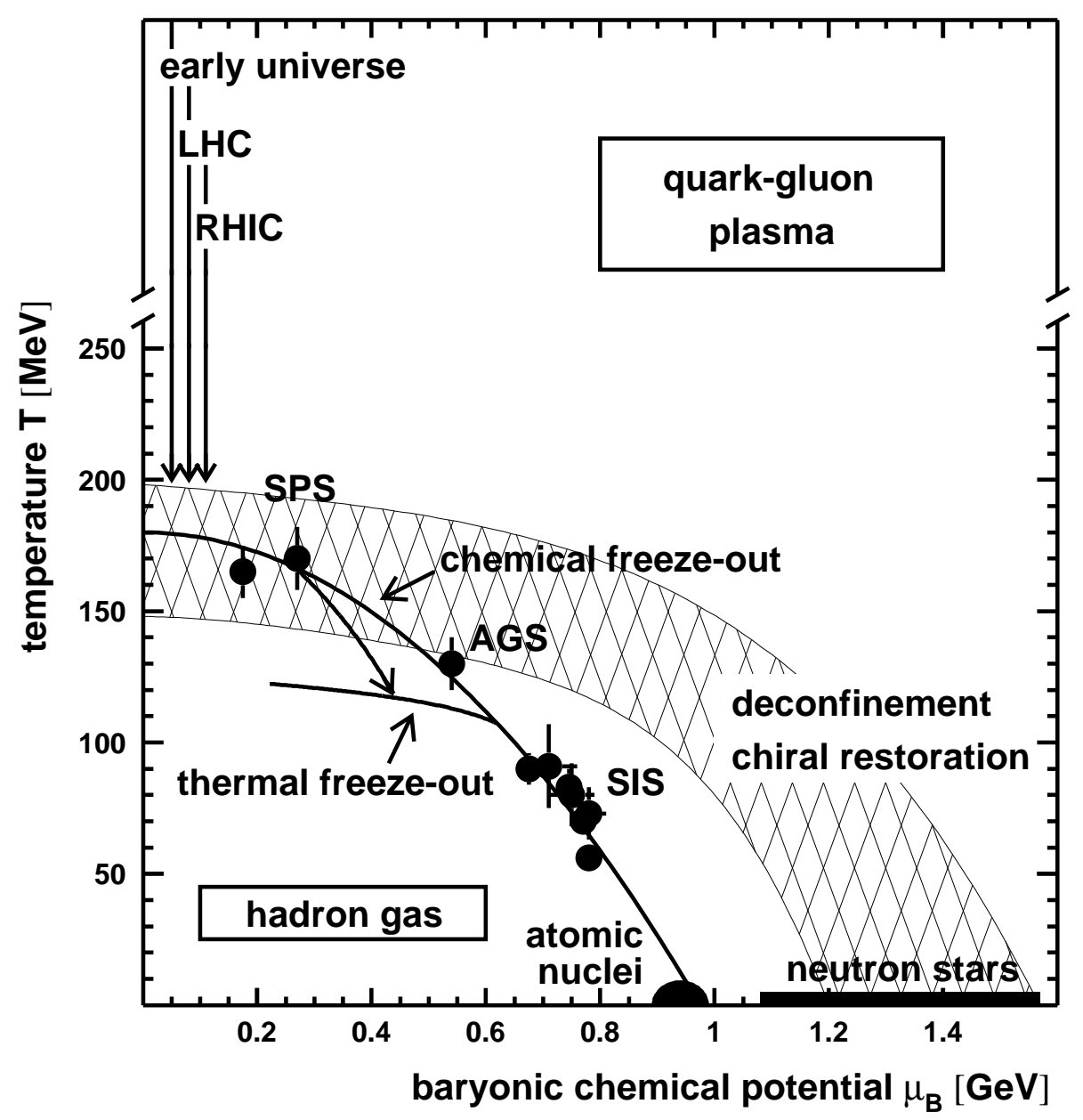

Figure 4. Phase diagram of hadronic matter and hadrochemical freeze-out points for heavy ion collisions at SIS, AGS and SPS. The hatched region indicates the expectation [9] for the phase boundary based on lattice QCD calculations at $\mu_{b}=0$. The arrow from chemical to thermal freeze-out curve for the SPS is calculated for expansion with constant entropy per baryon. Figure from [10,11.

instant in the systems evolution a unique combination of temperature and velocity (profile) is given. Using this approach it was shown [10 that a temperature of $160 \mathrm{MeV}$ cannot describe the $\mathrm{Pb}+\mathrm{Pb}$ SPS hadron spectra. Rather a temperature of $120 \mathrm{MeV}$ is required. The same temperature and velocity profile give a good description of the AGS Au+Au hadron spectra both in terms of overall slope and shape.

One clear exception from the simple scaling of spectral slopes with mass is visible in Fig. 5: the slope of the $\Omega$ spectrum is significantly lower than expected from the systematic trend although it is also much above a thermal distribution with $\mathrm{T}=120 \mathrm{MeV}$. This could be connected possibly with a smaller elastic cross section for the $\Omega$ or could conceivably also point to a different spatial distribution of the $\Omega$ 's in the fireball (at the inside the velocities are smaller). 
Information on the expansion of the fireball can also be gained from the two-particle correlations. The data can be sensitive both to the final volume as well as to the expansion dynamics.

Figure 6 shows the two-pion correlation function for positive and negative pions for central $\mathrm{Au}+\mathrm{Au}$ collisions at the AGS for the three components of relative momentum together with fits using a three-dimensional Gaussian parameterization (for details see [21]. Using the radius parameters in the three dimensions to estimate a lower limit for the volume at freeze-out one obtains $\mathrm{V}=2600 \mathrm{fm}^{3}$ or about twice the volume of the initial Au nucleus; clearly the system has expanded, in particular in view of the early compression (see below). The densities at thermal freeze-out for nucleons and pions given by this volume estimate are 0.11 and $0.12 / \mathrm{fm}^{3}$.

For $\mathrm{Pb}+\mathrm{Pb}$ collisions at the SPS the two-pion correlation function has been measured differentially as a function of the pair transverse momentum. In Figure 7 the (onedimensional) radius parameter $R_{x}$ is shown as a function of this variable for different rapidity bins. The decrease with increasing pair transverse momentum is a clear indication of the expansion of the system. The solid lines are fits assuming a certain expansion scenario [23] and temperature. Requiring at the same time a consistent description of the particle spectra an expansion velocity of about $50 \%$ of the speed-of-light and a 'true' standard deviation of the $\mathrm{x}$-distribution of $8.2 \mathrm{fm}$ are found together with a temperature of $120 \mathrm{MeV}$ from this analysis [22]. Note that this temperature agrees well with the one extracted [10] based only on spectra and a certain initial condition (see above). Using

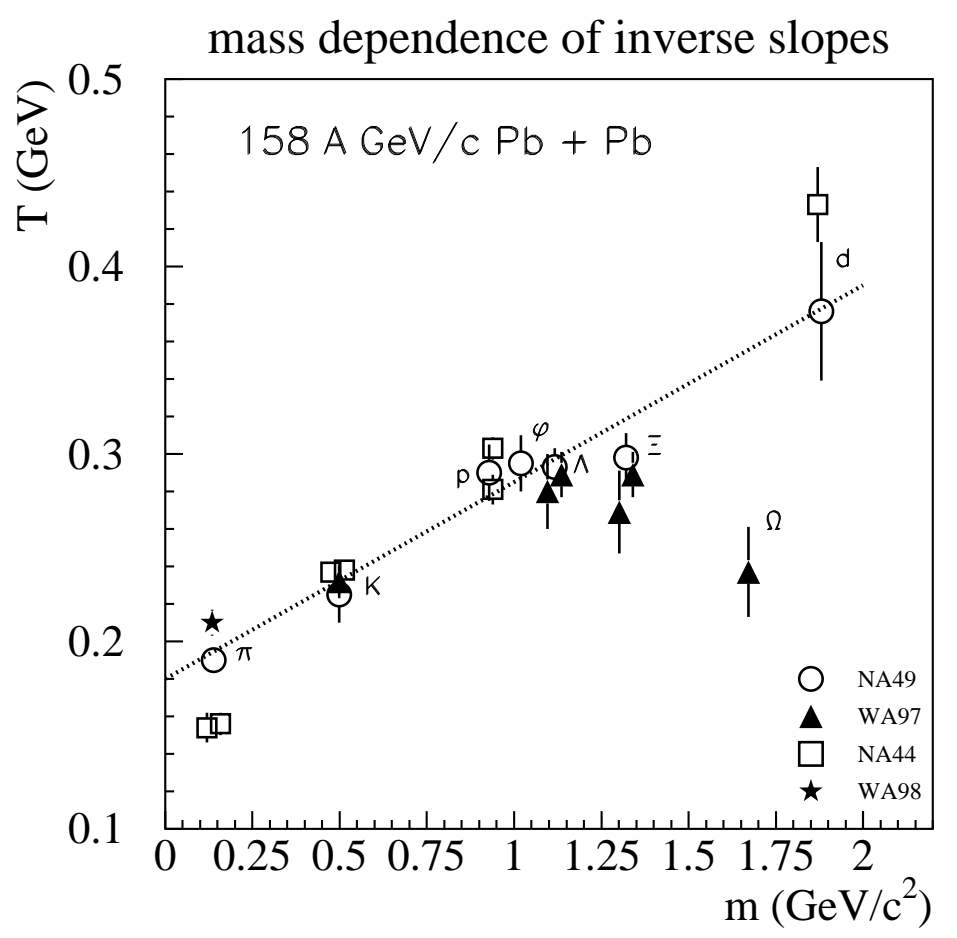

Figure 5. Systematics of inverse slope constants fitted to hadron spectra from central $\mathrm{Pb}+\mathrm{Pb}$ collisions at the SPS; experimental data from [14 17. 


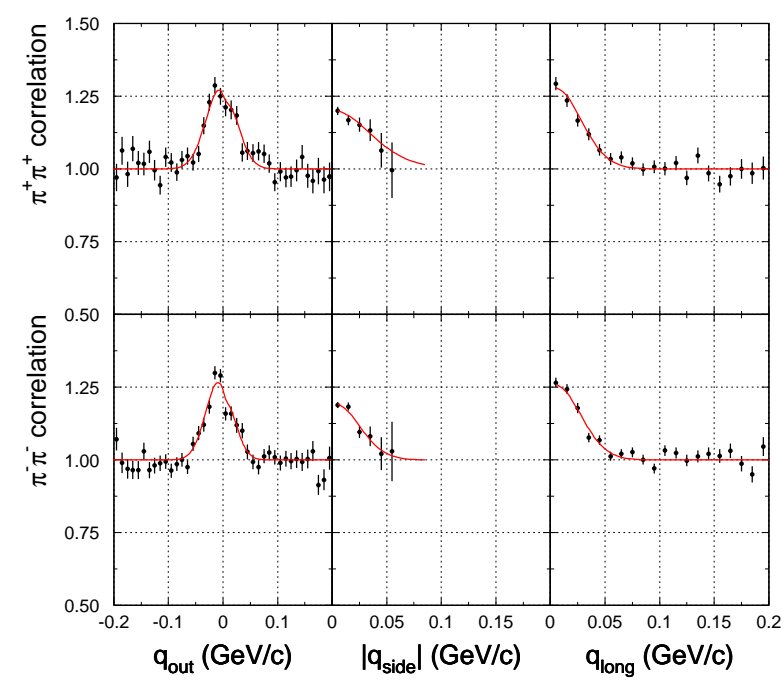

Figure 6. Two-pion correlation functions for central 10.8 A GeV/c Au+Au collisions at the AGS from E877. The correlation function is shown for the 3 relative momentum components and for positive and negative pions. Figure from 21] .

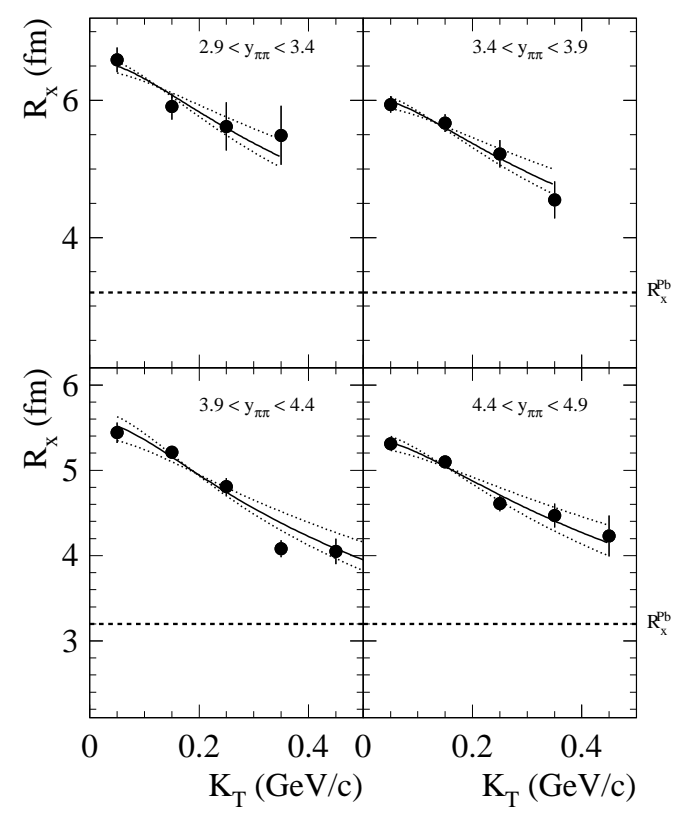

Figure 7. Transverse radius parameter as a function of the pion pair transverse momentum and in different rapidity bins for central $\mathrm{Pb}+\mathrm{Pb}$ collisions at the SPS measured by NA49. Also shown is a fit with an expanding thermal source model. Data and fits from [22]. The dashed line indicates the measured rms charge radius of $\mathrm{Pb}$ divided by square root of three.

the measured pion rapidity density and the transverse size information one determines a pion density of $0.12 / \mathrm{fm}^{3}$ at thermal freeze-out, a value identical to the one found for the AGS (see above).

The path from chemical to thermal freeze-out at the SPS is indicated in Fig. 1 by the arrow and it is defined by the final temperature and a constant entropy per baryon. At the AGS thermal and chemical freeze-out closely coincide.

\section{MEMORY OF THE INITIAL CONDITION}

Estimates for the initial baryon and energy density can be obtained from the rapidity distributions of protons and of transverse energy $E_{t}$. Figure 8 shows the distribution of protons in central $\mathrm{Au}+\mathrm{Au}$ collisions at the AGS; combining the data of two experiments the complete distribution is known. The distribution peaks at midrapidity indicating a very high degree of stopping. In [4 it was shown already that this distribution can be reproduced quantitatively assuming a uniform distribution of fireballs over one unit of rapidity in either direction from mid rapidity. This means the fastest fireballs are receding from the center with $76 \%$ of the speed-of-light, or the average longitudinal velocity is 0.46 c. Since this flat distribution corresponds to a Bjorken-type boost-invariant scenario (at least over a limited rapidity range) this same picture can be employed to estimate the 
early conditions, say at a time of $1 \mathrm{fm} / \mathrm{c}$ or $3 \cdot 10^{-24} \mathrm{~s}$. One finds a nucleon density of $1.1 / \mathrm{fm}^{3}$ - one order of magnitude above the density at thermal freeze-out - and an energy density of $1.4 \mathrm{GeV} / \mathrm{fm}^{3}$. The same type of analysis for SPS energies and $\mathrm{Pb}+\mathrm{Pb}$ collisions gives a baryon density of $0.65 / \mathrm{fm}^{3}$ and an energy density of about $3 \mathrm{GeV} / \mathrm{fm}^{3}$. At higher beam energy evidently the maximum baryon density is less and the maximum energy density is higher. Comparing to critical energy densities in state-of-the-art lattice QCD calculations the energy density at a the critical temperature is about $1 \mathrm{GeV} / \mathrm{cm}^{3}$. So based on the fact that the hadrochemical freeze-out points are on or close to the expected phase boundary and the estimate of the initially significantly higher energy and baryon densities it is likely that the system has indeed been beyond the phase boundary to the QGP at an early time.

Azimuthal distributions of transverse energy and of particle numbers, when analyzed relative to the reaction plane spanned by the impact parameter and the beam direction, show clear anisotropies for not too central collisions. There is a correlation between the final distributions at freeze-out and the impact parameter. Both a dipole moment, also called sideways flow, and a quadrupole moment, called elliptic flow have been found at the AGS and SPS for the heavy colliding systems. This is another link to the early conditions. I will pick here only one example and discuss the systematic dependence on beam energy of the elliptic flow, as it emerges from combining data from a number of experiments. The elliptic flow is quantified by the second Fourier coefficient of the azimuthal distribution $\mathrm{v}_{2}$ and the variation with beam energy is shown in Figure 9 for nucleons close to the c.m. rapidity.

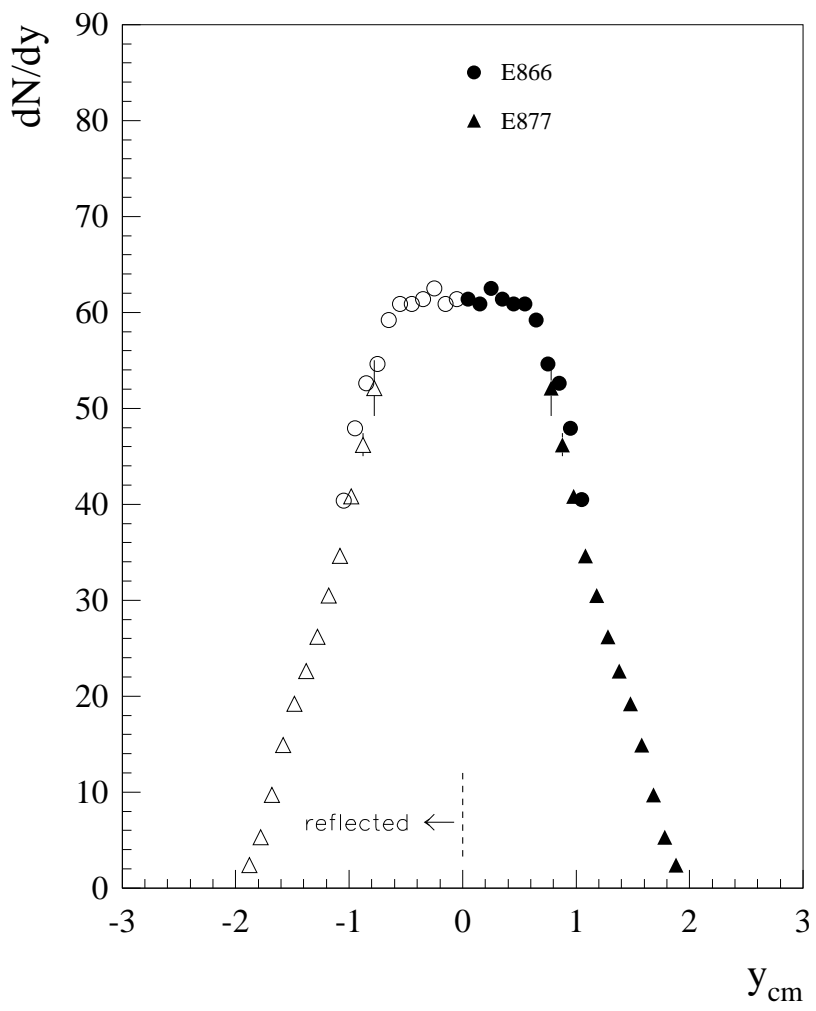

Figure 8. Proton rapidity density for central $\mathrm{Au}+\mathrm{Au}$ collisions at the AGS at $11 \mathrm{~A} \mathrm{GeV/c}$. Data from [24,25]. The open symbols are reflections of the measured points about mid rapidity. 


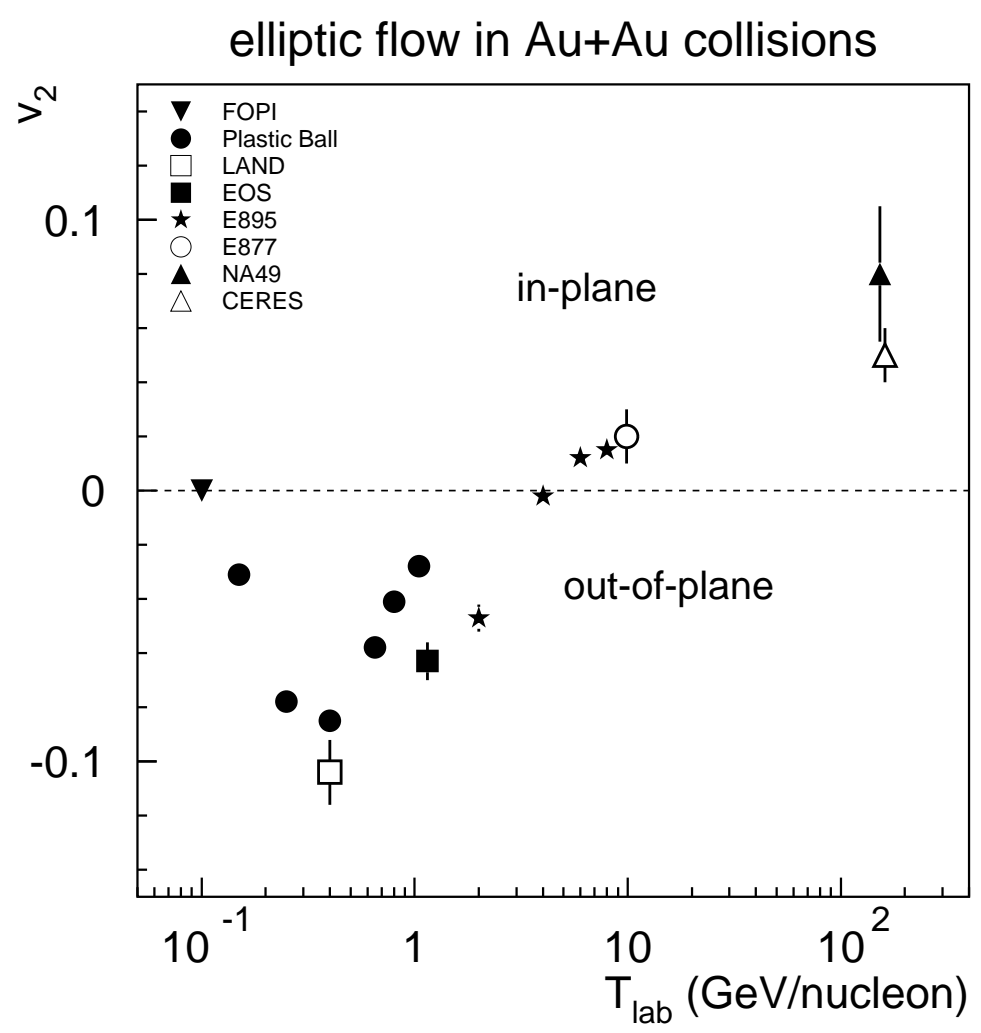

Figure 9. Dependence of the elliptic flow parameter $\mathrm{v}_{2}$, evaluated for nucleons near mid rapidity and for semi-central collisions, on the beam kinetic energy per nucleon. Data from [26 32]. Positive values mean preferential emission in the reaction plane.

A negative Fourier coefficient $\mathrm{v}_{2}$ means that the long axis of the ellipse is perpendicular to the reaction plane (out-of-plane elliptic flow) while for a positive coefficient it is in the plane (in-plane elliptic flow). The out-of-plane elliptic flow has been interpreted in terms of reinteractions of nucleons with projectile/target spectator nucleons. Naturally, as beam energy increases such remnants will eventually be too far away from mid-rapidity to affect the distribution of nucleons there and therefore one had expected this effect to decrease with increasing beam energy. In-plane elliptic flow develops due to the asymmetric shape of the overlap volume of the two nuclei and the related early pressure gradient. It has been predicted [33] that the elliptic flow is sensitive to the early pressure in the collision.

Even more recently, the beam energy dependence of the elliptic flow has been studied theoretically for different scenarios, including various types of equation of state and the possible influence of a phase transition [34]. A phase transition leads to an effective softening of the equation of state and therefore an upwardly steepening and then flattening S-curve with increasing beam energy. The very recent data from the E895 collaboration map the region between 2 and $8 \mathrm{GeV}$ and there is a close by point at $10 \mathrm{GeV}$ in good agreement (see Fig. 9). These data could just reflect the predicted [34] curvature due to a second order phase transition. The very rich data on flow, also of other particle species and as a function of transverse momentum and rapidity, deserve in this light full theoretical exploration, still missing at present. 


\section{VECTOR MESONS IN MEDIUM}

The decay of vector mesons into lepton pairs provides a means to look into the early, possibly even prehadronic, phase of the heavy ion collision since final state interactions of the leptons are negligible. First the low mass region with the $\rho, \omega, \phi$ mesons will be addressed.

The masses of vector mesons as a function of baryon density and temperature have been discussed in terms of their relation to the expected restoration of chiral symmetry. A measure for chiral symmetry breaking is the quark condensate which is found to drop to small values near the critical temperature in lattice QCD calculations [ [ 1 ]. How this restoration of chiral symmetry is reflected in observables has been a subject of much discussion (see e.g. [35]). QCD sum rules relate the quark condensate and the masses of vector mesons such as the $\rho$ or axial vectors such as the $a_{1}$, as first applied by [36]. This idea has led to the proposal of a linear downward scaling of the vector meson masses with increasing density, the so-called Brown-Rho scaling [37]. Later is was argued that the chiral partners such as the $\rho$ and $\mathrm{a}_{1}$ have to join but that this could happen at a nonzero mass determined by the value of the vector correlator in the hadronic medium (see discussion in [35]). A whole spectrum of theoretical possibilities has arisen for the masses and widths of vector mesons at finite density and temperature (see e.g. Table 3 in [38]). Clearly the situation calls for input from measurements.

An interesting opportunity stems from the short lifetime of the $\rho$ meson. Since it decays on the timescale of one or two $\mathrm{fm} / \mathrm{c}$ which is short as compared to the lifetime of the fireball it's decay samples the hot and dense medium and it's evolution as a function of time. Experimentally an enhancement as compared to expectations from $\mathrm{p}+\mathrm{A}$ collisions has been seen by the CERES collaboration in the dielectron spectrum in the mass region in between twice the pion mass and the $\rho, \omega$ pole for both $\mathrm{S}+\mathrm{Au}$ and $\mathrm{Pb}+\mathrm{Au}$ collisions [39,40] and for $\mathrm{S}+\mathrm{W}$ this is confirmed by the dimuon measurement from the HELIOS collaboration 41]. In 42 a high statistics measurement for $\mathrm{Pb}+\mathrm{Au}$ from CERES is added and the enhancement persists. As compared to simple expectations for hadron decay contributions the enhancement in the mass region $0.25-0.70 \mathrm{GeV} / \mathrm{c}^{2}$ is $42.6 \pm 0.5$ (stat.) \pm 0.5 (syst.). The enhancement is most significant at low pair transverse momenta and therefore Figure 10 shows the pair mass spectrum in two transverse momentum bins (see also Fig. 3 in [42]).

It has been argued that in the dense hadronic fireball processes such as pion annihilation could be an important source of dilepton pairs. Indeed this possibility is supported by the approximately quadratic scaling of the electron pair yield in this mass region [42]. However, calculations show that pion annihilation is dominated by the $\rho$ resonance and, while there is indeed a significant enhancement by this mechanism, the shape of the experimental spectrum is not reproduced at all. This is exemplified by the calculations shown as the solid lines in Fig. 10. Introducing in-medium modifications of the $\rho$ meson changes the situation drastically. One approach, coupling the $\rho$ meson to baryons [44, leads to a significant downward shift of the $\rho$ pole and a drastic increase in the width. This is the basis of the calculations shown as the dotted lines in Fig. 10 and it can be seen that now the experimental data can be accounted for. The effect of the Brown-Rho 
scaling is similar 45,43 although in detail there are differences. In order to differentiate between different possibilities and to also study the fate of the $\omega$ meson for which very interesting effects have been predicted as well [46] data with higher resolution and still higher statistics are required.

While the modifications of the $\rho$ and $\omega$ mesons address the state of the fireball between beginning hadronization and thermal freeze-out a much earlier time-scale is probed by the $\mathrm{J} / \Psi$ meson. The charm quark pairs are thought to be created in hard early collisions, in the kinematic regime relevant in present heavy ion experiments in gluon fusion. The question whether they hadronize into a $\mathrm{J} / \Psi$ or $\Psi^{\prime}$ meson is determined by the medium surrounding them. This study was fueled by the longstanding prediction by Satz and Matsui 47 that the heavy quark effective potential is screened by a high density of partons and thereby the $\mathrm{J} / \Psi$ and similar mesons melt in a process analogue to the Debye screening.

The production of $\mathrm{J} / \Psi$ mesons was studied from the beginning of the CERN heavy ion program. A suppression relative to the continuum was seen in $\mathrm{O}+\mathrm{Cu}, \mathrm{U}$ and $\mathrm{S}+\mathrm{U}$ collisions and this was complemented by a systematic study of $\mathrm{p}+$ nucleus collisions (a summary can be found in 48 ). The systematics of $\mathrm{J} / \Psi$ production in $\mathrm{p}+\mathrm{A}$ and $\mathrm{O}, \mathrm{S}+\mathrm{A}$ data can be summarized by a power law dependence of the $\mathrm{J} / \Psi$ production cross section as $(\mathrm{A} \cdot \mathrm{B})^{\alpha}$, where $\mathrm{A}$ and $\mathrm{B}$ are the mass numbers of target and projectile and $\alpha$ was determined as $0.911 \pm 0.016$.

In order to include measurements at various centralities for heavy ion collisions into the systematics a new variable $\mathrm{L}$ was introduced which measures the path length of nuclear matter traversed by the $\mathrm{J} / \Psi$ assuming a straight trajectory. The production cross section normalized to $\mathrm{A} \cdot \mathrm{B}$ was found to fall exponentially [49] as $\mathrm{A}(\mathrm{L})=\exp \left(-\rho L \sigma_{\text {abs }}\right)$ with $\sigma_{\text {abs }}$ of
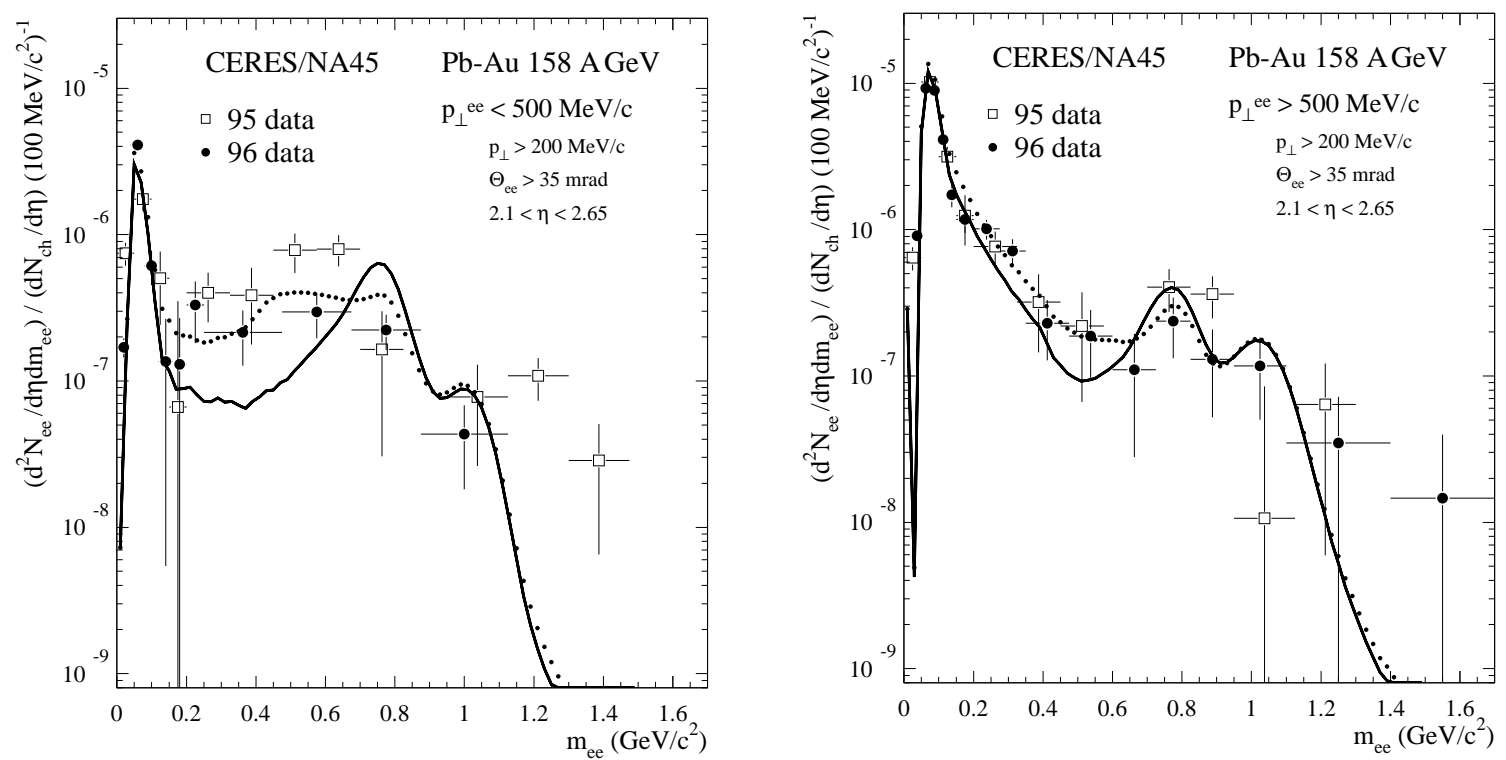

Figure 10. Invariant mass distributions of electron pairs for the most central third of the geometric cross section in $\mathrm{Pb}+\mathrm{Pb}$ collisions at $158 \mathrm{~A} \mathrm{GeV} / \mathrm{c}$ obtained by the CERES collaboration from the '95 and '96 data analyses, separated into samples with high and low pair $\mathrm{p}_{t}$ 42]. The data are compared to model calculations [43] based on the free $\rho$ properties (solid lines) and employing in-medium modifications of the $\rho$ (dotted lines). 


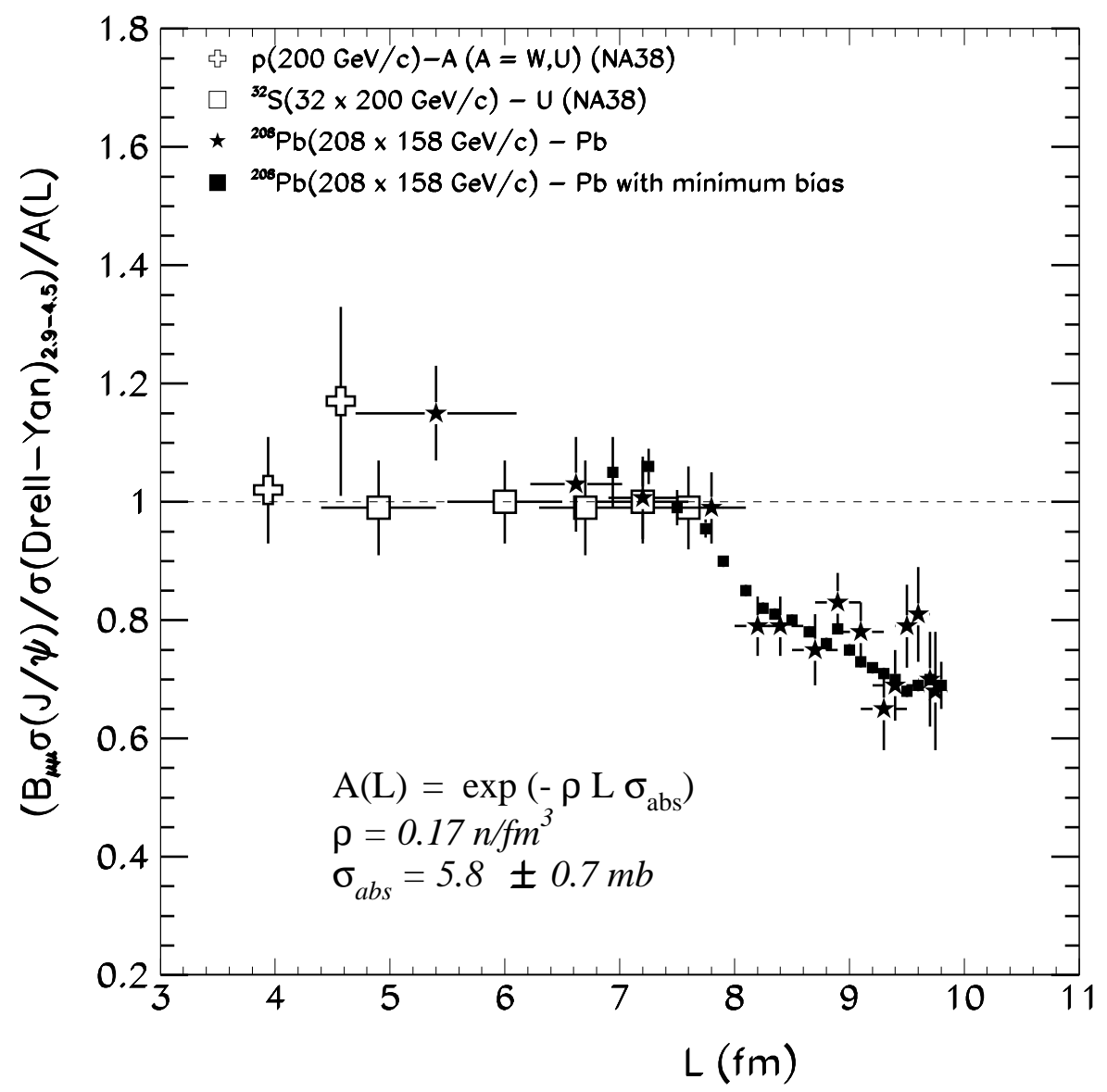

Figure 11. J/ $\Psi$ production normalized to the Drell-Yan yield as a function of the length $\mathrm{L}$ of nuclear matter traversed by the $\mathrm{J} / \Psi$. Figure from [51].

6-7 mb and the nuclear matter density $\rho=0.17 / \mathrm{fm}^{3}$. This surprizingly large cross section

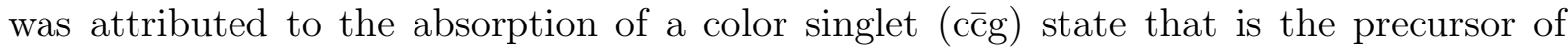
the fully formed hadron [50]. The data for $\mathrm{Pb}+\mathrm{Pb}$ collisions show a significant deviation from the scaling behavior just discussed. Figure 11 displays the cross section for $\mathrm{J} / \Psi$ production multiplied with the branching ratio for the dimuon decay and normalized to the Drell-Yan cross section divided by the exponential fall-off $\mathrm{A}(\mathrm{L})$.

The $\mathrm{Pb}+\mathrm{Pb}$ results are displayed for two different analyses as a function of centrality and for both one can see that the results for more peripheral collisions (smaller L) are close to the systematics of exponential fall-off obtained for lighter systems while the points for more central collisions (impact parameters below about $8 \mathrm{fm}$ ) exhibit an additional suppression. The stars represent data that are normalized to the Drell-Yan cross section measured in the same experiment and the statistical errors are dominated by the much smaller Drell-Yan cross section. The squares avoid this statistical uncertainly by instead utilizing a measured minimum bias transverse energy distribution and a calculated ratio of Drell-Yan to minimum bias cross section for every centrality (or $\mathrm{E}_{\mathrm{t}}$ ). Again a systematic drop is visible for b below $8 \mathrm{fm}$ (L above $8 \mathrm{fm}$ ). Comparison of the two analyses establishes that the drop can only be due to a reduced $\mathrm{J} / \Psi$ cross section.

Since the drop in $\mathrm{J} / \Psi$ production appears at a certain centrality in $\mathrm{Pb}+\mathrm{Pb}$ collisions it 
is indeed plausible that this is related to a certain critical energy density, possibly reached over a certain critical volume. It has been demonstrated recently [52] in a parton cascade calculations that at SPS energy and a time of $0.3 \mathrm{fm} / \mathrm{c}$ the energy density in the center of the colliding nuclei exceeds $9 \mathrm{GeV} / \mathrm{fm}^{3}$ while in $\mathrm{S}+\mathrm{S}$ collisions a value of $3 \mathrm{GeV} / \mathrm{fm}^{3}$ is never exceeded. This idea has been exploited in a model [53,54 where formation of QGP bubbles is assumed and above a critical density of $1.4 \mathrm{GeV} / \mathrm{fm}^{3}$ and a radius of $4 \mathrm{fm}$ all $\chi_{c}$ are dissociated; the $40 \%$ portion of $\mathrm{J} / \Psi$ that is produced via decays of the $\chi_{c}$ thus disappears. At higher energy densities also the $\mathrm{J} / \Psi$ itself is expected to melt in the plasma. This model still requires some ad hoc assumptions (see above) and any alternative solution needs to be excluded. On the other hand there is now a body of high quality data for different systems and centralities including the transverse momentum distribution of the $\mathrm{J} / \Psi$ mesons [55] and any theoretical interpretation needs to address in a consistent way all available data.

\section{OUTLOOK}

Present fixed target experiments at the AGS and SPS studying collisions between the heaviest nuclei exhibit a number of interesting features. The hadron yields are frozen at or very near the expected phase boundary to the QGP. At this point strangeness is, contrary to $\mathrm{p}+\mathrm{p}$ collisions, also in equilibrium, i.e. saturated, even for rarely produced multiply strange hadrons. It is not clear how this equilibration, also of particle species which are expected to have small production cross sections, can come about in a purely hadronic scenario and on the time scales available in this case. This, together with the circumstantial evidence that the initial baryon and energy density at AGS and SPS are beyond the estimated critical values early in the collision, makes it likely that indeed the system is in the QGP phase for some time before it hadronizes again. Estimates of the maximum temperature reached give values of about $200 \mathrm{MeV}$, i.e. not much beyond the critical temperature. This could still result in a visible signal in direct photons radiated during the evolution if the hadronization takes long enough. There is tantalizing first indication that there may indeed be a signal [56]. The anisotropic flow and in particular the elliptic flow and their beam energy dependence are sensitive to the equation of state and changes in it. The values around the zero crossing at the AGS could be related to a softening of the equation of state associated with a transition from hard hadronic matter to softer quark matter. The enhancement in the dilepton mass spectrum above twice the $\pi$ and below the $\rho$ mass indicates in-medium modifications of the $\rho$ meson. Their relation to chiral symmetry restoration and possible modifications of the other light vector mesons need more experimental and theoretical study. The unexpected additional suppression of the $\mathrm{J} / \Psi$ for more central $\mathrm{Pb}+\mathrm{Pb}$ collisions as compared to previously studied $\mathrm{p}+\mathrm{p}$, $\mathrm{p}+\mathrm{A}$, and $\mathrm{A}+\mathrm{B}$ systems could indicate the predicted color screening in the QGP. But a consistent implementation of other possible scenarios and a comparison to all availalbe data is still needed to see whether this is the only explanation of this intriguing signal.

The advent of the colliders RHIC at Brookhaven later this year and of the LHC in 2005 will add a new dimension to the study of the QGP. At these accelerators collisions of the heaviest nuclei will be studied at c.m. energies a factor of 12 and 330 higher than presently available at the SPS. Based on the new information on parton distributions in 


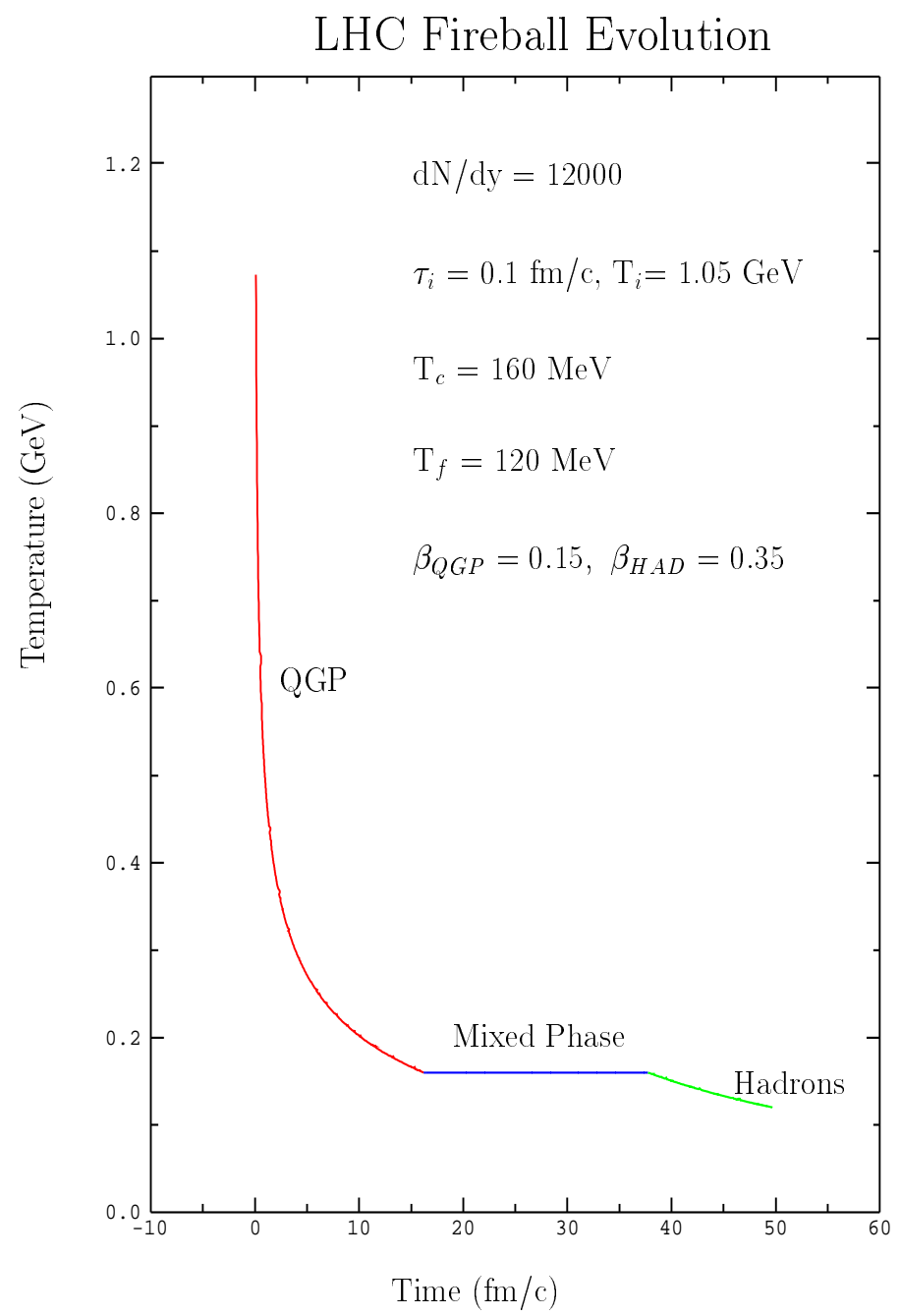

Figure 12. Expected evolution of the fireball after a $\mathrm{Pb}+\mathrm{Pb}$ collision at LHC. Figure from [57].

the nucleon from HERA this leads to a large number of partons in the early phase and to a rapid thermal equilibration. It is estimated that there will be about 4000 gluons per unit rapidity above $\mathrm{p}_{t}=2 \mathrm{GeV} / \mathrm{c}$ and about a factor of ten less quarks leading to very copious (mini)jet formation. This implies that the system equilibrates thermally on the timescale of $0.1 \mathrm{fm} / \mathrm{c}$ and would lead to initial temperatures of 450 and $1000 \mathrm{MeV}$ at RHIC and LHC, respectively. Both values are well beyond the critical temperature and should allow to study the (ideal) parton gas and it's evolution and eventual hadronization.

A typical expectation for the evolution of the system after the initial short equilibration phase is shown in Figure 12 for a $\mathrm{Pb}+\mathrm{Pb}$ collision at the LHC. With the somewhat conservative expectation that transverse expansion, and therefore more rapid cooling, is already significant in the plasma phase the plasma expands and cools to the critical temperature over about 10-15 fm/c [57]. Then hadronization takes place and since a large number of degrees of freedom has to be converted into a smaller number corresponding to a hot hadron gas the volume has to grow accordingly (so that entropy stays at least constant). This takes typically $20 \mathrm{fm} / \mathrm{c}$. The final expansion of the hadronized system until thermal freeze-out is presumably similar to what is currently already seen at the 
SPS and this final point could again be determined by the pion density of $0.1 / \mathrm{fm}^{3}$. With the expected very large pion multiplicities (about 8000 per unit rapidity at LHC) this leads also to a very huge volume in the final state.

With the very large initial temperatures and the clearly partonic nature of the early state new signatures and tools become available. A comprehensive discussion is beyond the scope of the present discussion. Among the interesting features to be studied are the energy loss of partons in a QGP, the associated question of jet quenching, direct thermal radiation of the very hot plasma via $q \bar{q}$ annihilation and the QCD Compton process, color screening of $\mathrm{J} / \Psi, \Psi^{\prime}$, and $\Upsilon$ states, open charm and beauty production, in addition to the array of phenomena studied already presently at the lower energies.

\section{REFERENCES}

1. See e.g. the proceedings of the Lattice'98 and preceeding conferences, Nucl. Phys. B63 (1998).

2. C. Bernard et al., MILC collaboration, Phys. Rev. D56 (1997) 5584.

3. E. Laermann, Nucl. Phys. B63 (1998) 114.

4. J. Stachel, Nucl. Phys. A610 (1996) 509c.

5. P. Braun-Munzinger, J. Stachel, J.P. Wessels, N. Xu, Phys. Lett. B344 (1994) 43.

6. E. Andersen et al., WA97 collaboration, preprint CERN-EP/99-29, Phys. Lett. B in print.

7. P. Braun-Munzinger, I. Heppe, J. Stachel, preprint nucl-th/9903010 and subm. Phys. Lett. B.

8. F. Becattini, J. Phys. G23 (1997) 1933.

9. P. Braun-Munzinger and J. Stachel, Nucl. Phys. A606 (1996) 320.

10. P. Braun-Munzinger and J. Stachel, Nucl. Phys. A638 (1998) 3c.

11. Modifications to Fig. 4 courtesy of Ralf Averbeck.

12. E. Laermann, Nucl. Phys. A610 (1996) 1c.

13. J. Cleymans and K. Redlich, GSI preprint-98-43.

14. I.G. Bearden et al., NA44 collaboration, Phys. Rev. Lett. 78 (1997) 2080; M. Kaneta for the NA44 collaboration, Nucl. Phys. A638 (1998) 419c; A. Sakaguchi for the NA44 collaboration, ibidum 103c.

15. G. Roland for the NA49 collaboration, Nucl. Phys. A638 (1998) 91c; F. Pühlhofer for the NA49 collaboration, ibidum 431c; H. Appelshäuser et al., NA49 collaboration, Phys. Lett. B444 (1998) 523.

16. E. Andersen et al., WA97 collaboration, Phys. Lett. B433 (1998) 209; R. Lietava for the WA97 collaboration in Proc. Strangeness 98, J. Phys. G: Nucl. Part. Phys. 25 (1999).

17. M.M. Aggarwal et al., WA98 collaboration, Nucl. Phys. A638 (1998) 147c.

18. One of the earliest references where this connection is made is P.J. Siemens and J.O. Rasmussen, Phys. Rev. Lett. 42 (1979) 880.

19. P. Braun-Munzinger, J. Stachel, J.P. Wessels, N. Xu, Phys. Lett. B365 (1996) 1.

20. S. Esumi, S. Chapman, H. van Hecke, N. Xu, Phys. Rev. C55 (1997) R1.

21. J. Barrette et al., E877 collaboration, Phys. Rev. Lett. 78 (1997) 2916.

22. H. Appelshäuser et al., NA49 collaboration, Eur. Phys. J. C2 (1998) 661. 
23. S. Chapman, J.R. Nix, U. Heinz, Phys. Rev. C52 (1995) 2694.

24. R. Lacasse, Ph.D. thesis McGill University, 1998; J. Barrette et al., E877 collaboration, manuscript in preparation.

25. L. Ahle et al., E866 collaboration, Phys. Rev. C57 (1998) R466.

26. Bastid et al., FOPI collaboration, Nucl. Phys. A622 (1997) 573.

27. H.H. Gutbrod, K.H. Kampert, B. Kolb, A.M. Poskanzer, H.G. Ritter, R. Schicker, H.R. Schmidt, Phys. Rev. C42 (1990) 640.

28. Y. Leifels et al., LAND collaboration, Phys. Rev. Lett. 71 (1993) 963.

29. C. Pinkenburg et al., E895 collaboration, subm. Phys. Rev. Lett. (1999).

30. J. Barrette et al., E877 collaboration, Phys. Rev. C55 (1997) 1420.

31. H. Appelshäuser et al., NA49 collaboration, Phys. Rev. Lett. 80 (1998) 4136.

32. F. Ceretto for the CERES collaboration, Nucl. Phys. A638 (1998) 467c.

33. H. Sorge, Phys. Rev. Lett. 78 (1997) 2309.

34. P. Danielewicz et al., Phys. Rev. Lett. 81 (1998) 2438.

35. J. Wambach and R. Rapp, Nucl. Phys. A638 (1998) 171c.

36. T. Hatsuda and S.H. Lee, Phys. Rev. C52 (1992) R34.

37. G.E. Brown and M. Rho, Phys. Rev. Lett. 66 (1991) 2720.

38. S.H. Lee, Nucl. Phys. A638 (1998) 183c.

39. G. Agakichiev et al., CERES collaboration, Phys. Rev. Lett. 75 (1995) 1272.

40. G. Agakichiev et al., CERES collaboration, Phys. Lett. B422 (1998) 405.

41. M. Masera for the HELIOS collaboration, Nucl. Phys. A590 (1995) 93c.

42. B. Lenkeit for the CERES collaboration, these proceedings.

43. R. Rapp, proceedings Rencontres de Moriond '98, nucl-th/9804065; R. Rapp and C. Gale, hep-ph/9902268; and R. Rapp, private communication.

44. R. Rapp, G. Chanfray, J. Wambach, Nucl. Phys. A617 (1997) 472.

45. G.E. Brown, G.Q. Li, R. Rapp, M. Rho, J. Wambach, Acta Phys. Polon. B29 (1998) 2309.

46. F. Klingl, N. Kaiser, W. Weise, Z. Phys. A356 (1996) 193; Nucl. Phys. A624 (1997) 527.

47. T. Matsui and H. Satz, Phys. Lett. B178 (1986) 416.

48. C. Lourenco, Nucl. Phys. A610 (1996) 552c.

49. C. Gerschel and J. Hüfner, Nucl. Phys. A544 (1992) 513c.

50. D. Kharzeev and H. Satz, Phys. Lett B366 (1996) 316.

51. M.C. Abreu et al., NA50 collaboration, preprint CERN-EP/99-13 and Phys. Lett. B (1999) in print.

52. D.K. Srivastava and K. Geiger, Nucl. Phys. A647 (1999) 136.

53. D. Kharzeev, Nucl. Phys. A638 (1998) 279c.

54. D. Kharzeev, M. Nardi, H. Satz, preprint hep-ph/9707308.

55. N.S. Topilskaya for the NA50 collaboration, these proceedings.

56. V. Manko for the WA98 collaboration, these proceedings.

57. P. Braun-Munzinger, private communication. 